\title{
The millipedes of Barrow Island, Western Australia (Diplopoda)
}

\author{
Catherine A. Car' ${ }^{1}$ Megan Short ${ }^{2}$, Cuong Huynh² and Mark S. Harvey ${ }^{1,3,4,5,6}$ \\ ${ }^{1}$ Department of Terrestrial Zoology, Western Australian Museum, Locked Bag 49, Welshpool DC, \\ Western Australia 6986, Australia. \\ 2 Deakin University, 221 Burwood Hwy, Burwood, Victoria 3125, Australia. \\ ${ }^{3}$ Research Associate: Division of Invertebrate Zoology, American Museum of Natural History, 79th \\ Street @ Central Park West, New York, New York 10024-5192, USA. \\ ${ }^{4}$ Department of Entomology, California Academy of Sciences, Golden Gate Park, San Francisco, \\ California 94103-3009 USA. \\ ${ }^{5}$ School of Animal Biology, University of Western Australia, Crawley, \\ Western Australia 6009, Australia. \\ ${ }^{6}$ Adjunct Professor: School of Natural Sciences, Edith Cowan University, Joondalup, \\ Western Australia 6027, Australia.
}

\begin{abstract}
Six species of millipedes are recorded from Barrow Island, including three species of pin-cushion millipedes of the order Polyxenida, Lophoturus madecassus (Marquet and Condé, 1950) (Lophoproctidae), Unixenus mjoebergi (Verhoeff, 1924) (Polyxenidae) and Phryssonotus novaehollandiae (Silvestri, 1923) (Synxenidae), a single species of the order Spirobolida, Speleostrophus nesiotes Hoffman, 1994 (Trigoniulidae), and two species of the order Polydesmida, Boreohesperus dubitalis Car and Harvey, 2013 (Paradoxosomatidae) and one species of the family Haplodesmidae (genus and species indet.). Lophoturus madecassus is circum-tropical in distribution, Unixenus mjoebergi and Phryssonotus novaehollandiae are found also on mainland Australia, but the other three species are endemic to the island. Speleostrophus nesiotes is a highly modified troglobiotic species, currently listed as threatened by the Western Australian government. It is unclear at present whether the haplodesmid specimen is a troglobite.
\end{abstract}

KEYWORDS: short-range endemics, biodiversity, threatened species

\section{INTRODUCTION}

Barrow Island lies ca. $55 \mathrm{~km}$ from the northwest coast of Western Australia at ca. $20^{\circ} 45^{\prime} \mathrm{S}$, $115^{\circ} 25^{\prime} \mathrm{E}$. It is the largest off-shore island in Australia, and home to many unique biotic elements and the last refuge of several mammal species that have otherwise been extirpated on the mainland by introduced carnivores. The superficial geology of the island is predominantly comprised of sedimentary deposits which have formed sandstones (Dept. of Resources, Energy and Tourism 2010) as well as large areas of limestone karst which support a unique subterranean faunal community (Humphreys 2000, 2001). Barrow Island lies within the North Coast IBRA (Interim Biogeographic Regionalisation for Australia) region
(Thackway and Cresswell 1995; Environment Australia 2000) and has many biogeographical links with the neighbouring Pilbara bioregion and with the Cape Range which is situated ca. $155 \mathrm{~km}$ to the south-west.

Six species of millipedes have been identified from Barrow Island, based upon numerous collections made by different organisations since the 1980s. We present a synopsis of the fauna of the island. Four of the six species have been previously named, but the fifth represents a new species of Boreohesperus Shear, 1992, B. dubitalis sp. nov. and is described in a recent paper (Car and Harvey 2013). The remaining species is unidentified at present, but its presence on the island is noted here. 


\section{MATERIAL AND METHODS}

The specimens examined for this study are lodged in the Western Australian Museum, Perth (WAM), and were examined with Leica MZ6 and MZ16A stereo microscopes. The images were generated with a Leica MZ16A automontage imaging system. To confirm the identification of $L$. madecassus (Marquet and Condé 1950) 2 specimens (from collections T104047 and T110379) were cleared in $15 \%$ potassium hydroxide, heated in a water-bath for 2 minutes at $80^{\circ} \mathrm{C}$, neutralised in $20 \%$ acetic acid for 2 minutes, rinsed in distilled water and dehydrated in a series of ethanol baths prior to staining with 1\% Fast Green solution to increase contrast. The head and body were separated, the body cut open with a single latero-longitudinal incision and contents removed. After rinsing in $100 \%$ ethanol, stained specimens were transferred to $100 \%$ isopropanol, then to xylene and mounted on slides with DPX synthetic resin and examined using an Olympus Vanox compound microscope. The maps were prepared with ArcGIS version 9.3. The taxonomic data and literature were partly obtained from Mesibov (2013).

\section{TAXONOMY}

\section{Subclass Penicillata Latreille, 1831 \\ Order Polyxenida Lucas, 1840}

\section{Family Lophoproctidae Silvestri, 1897}

\section{Genus Lophoturus Brolemann, 1931}

Lophoproctus (Lophoturus) Brolemann, 1931: 303-304.

Alloproctus Silvestri, 1948: 219-220 (junior homonym of Alloproctus Bergroth, 1924).

Oroxenus Chamberlin, 1955: 47.

Alloproctinus Jeekel, 1963: 156 (replacement name for Alloproctus Silvestri, 1948).

\section{TYPE SPECIES}

Lophoproctus (Lophoturus) obscurus Brolemann, 1931, by original designation.

\section{REMARKS}

Lophoturus was originally described as a subgenus of Lophoproctus but was raised to genus rank by Condé and Nguyen Duy-Jacquemin (1977). The genus Lophoturus is found globally with 23 described species (Nguyen Duy-Jacquemin and Geoffroy 2003).

\section{Lophoturus madecassus \\ (Marquet and Condé, 1950)}

Alloproctus madecassus Marquet and Condé, 1950: 126-128, Figures 3-4.

Lophoturus madecassus (Marquet and Condé): Condé and Nguyen Duy-Jacquemin, 1977: 909-915.

\section{MATERIAL EXAMINED}

Australia: Western Australia: Barrow Island: 1 individual, Bore S9P2T1-2, 2049'12"S. 115 21'04" E, troglofauna trap, 14 June 2010, J. Alexander and J. Cairnes (WAM T1040047); 2 individuals, 2047'32"S, $115^{\circ} 26^{\prime} 53^{\prime \prime} \mathrm{E}$, troglofauna trap $10 \mathrm{~m}, 17$ January 2010, J. Alexander (WAM T110379); 2 individuals, $20^{\circ} 48^{\prime} 47^{\prime \prime} \mathrm{S}, 115^{\circ} 25^{\prime} 33^{\prime \prime} \mathrm{E}$, troglofauna trap, $8 \mathrm{~m}, 17$ January 2010, J. Alexander (WAM T110380).

\section{DIAGNOSIS}

Lophoturus madecassus can be distinguished from other species of the genus by the presence in adults of 11 pairs of legs (Figure 1).

\section{DESCRIPTION}

See Marquet and Condé (1950), and Nguyen DuyJacquemin and Condé (1982).

\section{REMARKS}

Lophoturus madecassus was first described as a species of Alloproctus by Marquet and Condé (1950) based on 3 adult female specimens collected from a park in the town of Tuléar (Toliara) Madagascar. The species was redescribed by Nguyen DuyJacquemin and Condé (1982) based on 1 adult male, 8 adult females and 12 immature stadia collected from tropical forest litter on the island of Nomuka Iki, Tonga and palm forest litter on the Atoll of Suvorov, Cook Islands. The species has also been recorded from southern Algeria, Ivory Coast, Jamaica, Florida (Nguyen Duy-Jacquemin and Condé 1982), and Cape Verde Islands (Enghoff 1993) indicating a circum-tropical distribution. Hoffman (1999) suggested that the species has been synanthropically dispersed, although MS considers dispersal by birds more probable, with the tiny millipedes being carried entangled by their bristles in feathers.

It is of interest that the examples of the species on Barrow Island were collected at some depth from the surface. Previous collections of the species have all been from litter but species of the family Lophoproctidae are known to frequent caves and to seek protection in crevices in adverse conditions.

This is the first record of L. madecassus from Australia (Figure 7). 


\section{Family Polyxenidae Lucas, 1840}

\section{Unixenus Jones, 1944}

Monoxenus Jones, 1937: 138 (junior homonym of Monoxenus Kolbe, 1893).

Unixenus Jones, 1944: 94 (replacement name for Monoxenus Jones, 1937).

\section{TYPE SPECIES}

Monoxenus padmanabhii Jones, 1937, by monotypy.

\section{REMARKS}

The polyxenid genus Unixenus is widespread and represented by 12 species in Africa, Madagascar, India and Australia (Nguyen Duy-Jacquemin and Geoffroy 2003; Short and Huynh 2011, 2013).
Eight of these species are restricted to Australia: $U$. attemsi Nguyen Duy-Jacquemin and Condé, 1967, U. barrabaensis Short and Huynh, 2013, $U$. carnarvonensis Short and Huynh, 2013, U. corringlensis Short and Huynh, 2013, U. corticolus Short and Huynh, 2011, U. karajinensis Short and Huynh, 2011, U. mjoebergi (Verhoeff, 1924) and U. myallensis Short and Huynh, 2013 (Short and Huynh 2011, 2013).

\section{Unixenus mjoebergi (Verhoeff, 1924)}

Monographis mjoebergi Verhoeff, 1924: 38 [as Monographis mjöbergi].

\section{MATERIAL EXAMINED}

Australia: Western Australia: Barrow Island: Site 17, 20 $47^{\prime} 38^{\prime \prime} \mathrm{S}, 115^{\circ} 27^{\prime} 24^{\prime \prime} \mathrm{E}, 10$ individuals, post
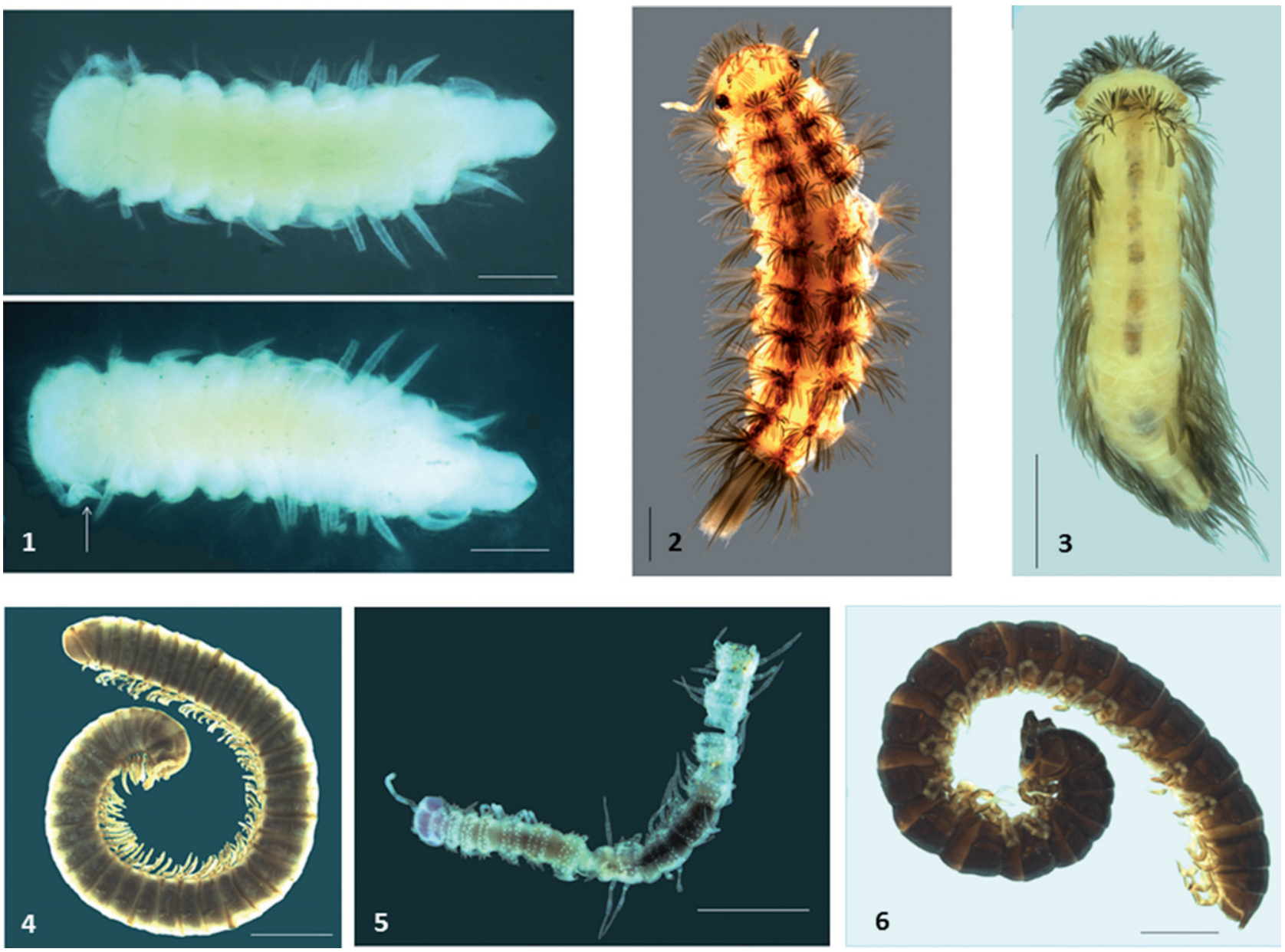

FIGURES 1-6

The millipedes on Barrow Island: 1, Lophoturus madecassus (Marquet and Condé, 1950) (WAM T110380), top, dorsal view; bottom, ventral view, showing antenna (arrow); 2, Unixenus mjoebergi (Verhoeff, 1924) (WAM T107716); 3, Phryssonotus novaehollandiae (Silvestri, 1923) (WAM T114011); 4, Speleostrophus nesiotes Hoffman, 1994 (WAM T76951); 5, Haplodesmidae (genus and species indet.) (WAM T116231); 6, Boreohesperus dubitalis Car and Harvey, 2013 (WAM T123094). Scale bar = $0.2 \mathrm{~mm}$ (Figure 1), $0.5 \mathrm{~mm}$ (Figures 2, 3), $2 \mathrm{~mm}$ (Figures 4, 5), $1 \mathrm{~mm}$ (Figure 6) 

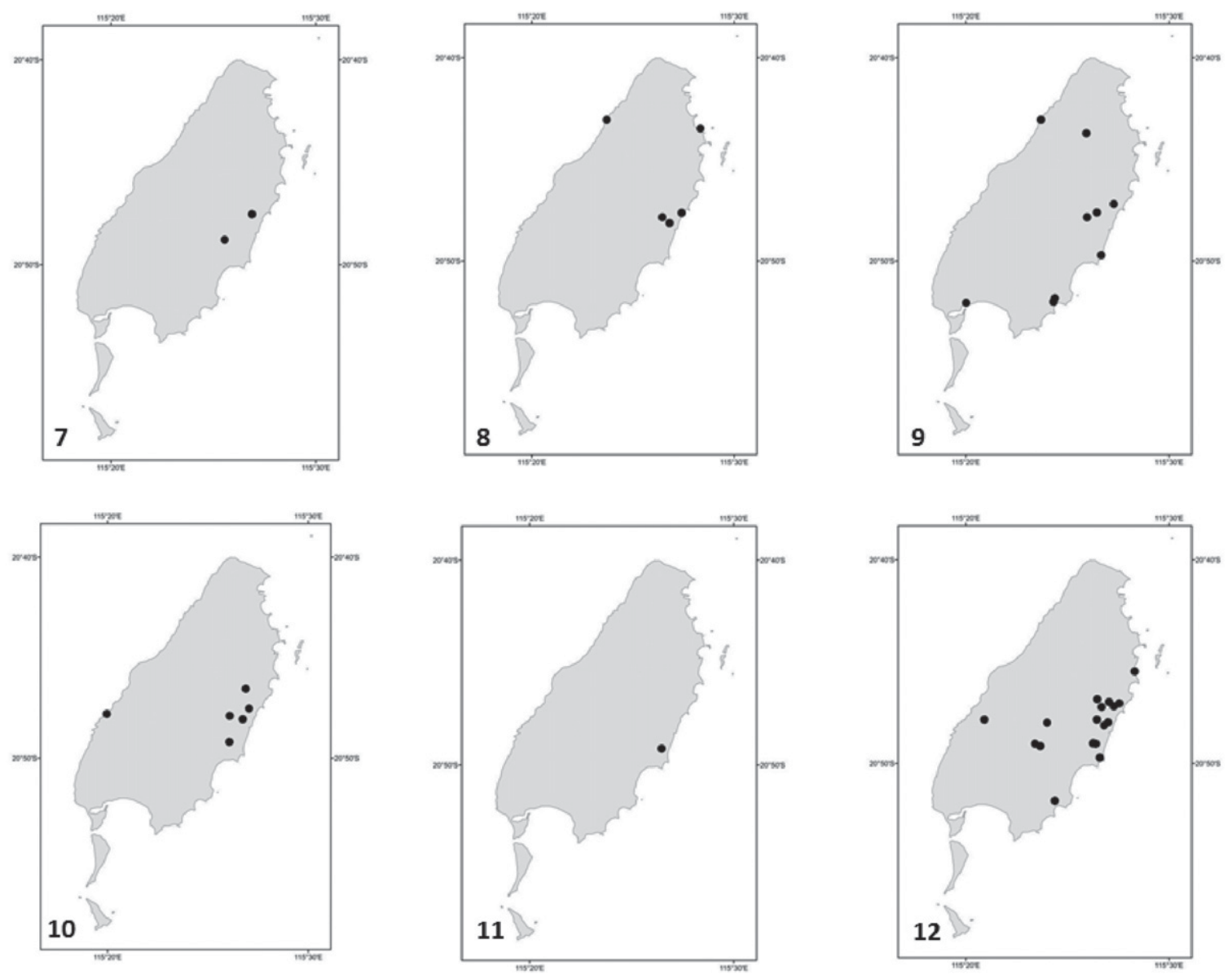

FIGURES 7-12 Maps showing recorded distributions of millipedes on Barrow Island: 7, Lophoturus madecassus (Marquet and Condé, 1950); 8, Unixenus mjoebergi (Verhoeff, 1924); 9, Phryssonotus novaehollandiae (Silvestri, 1923); 10, Speleostrophus nesiotes Hoffman, 1994; 11, Haplodesmidae (genus and species indet.); 12, Boreohesperus dubitalis Car and Harvey, 2013.

Winkler sac litter, 17 May 2005, S. Callan et al. (WAM T71112); 100+ individuals, suction samples, 24 April 2005, K. Edward and S. Callan (WAM T71114, T71115, T129331); 8 individuals, suction sample 06, 24 April 2005, K. Edward and S. Callan (WAM T126121); 15 individuals, suction sample 7, 24 April 2005, K. Edward and S. Callan (WAM T129453); 4 individuals, wet pitfalls, 24 April 2005-4 May 2005, K. Edward and S. Callan (WAM T129316, T129318); 8 individuals, wet pitfalls, 17-27 May 2005, S. Callan et al. (WAM T129319); 5 individuals, wet pitfalls, 24-29 April 2005, K. Edward and S. Callan (WAM T129323, T129451); 5 individuals, Winkler sac, 17 May 2005, S. Callan et al. (WAM T129328); Site $105,20^{\circ} 48^{\prime} 08^{\prime \prime} \mathrm{S}, 115^{\circ} 26^{\prime} 48^{\prime \prime} \mathrm{E}$, 50+ individuals, suction samples (combined), 24 April 2005, K. Edward and S. Callan (WAM T71111); 50+ individuals, Winkler sac samples, 24 April 2005, K. Edward and S. Callan (WAM T71113); 1 individual, wet pitfall traps, 17-22 May 2005, S. Callan et al.
(WAM T129452); 1 individual, hand sorted litter, 24 April 2005, K. Edward and S. Callan (WAM T129314); 1 individual, Winkler sac litter, 17 May 2005, S. Callan et al. (WAM T129317); 1 individual, Cape Malouet, $20^{\circ} 43^{\prime} 03^{\prime \prime} \mathrm{S}, 115^{\circ} 23^{\prime} 42^{\prime \prime} \mathrm{E}$, under rocks on cliff face, 5 November 1993, M.S. Harvey and J.M. Waldock (WAM T71145); Chevron Texaco camp, 20 $0^{\circ} 49^{\prime} 43^{\prime \prime} \mathrm{S}, 115^{\circ} 26^{\prime} 36^{\prime \prime} \mathrm{E}, 3$ individuals, suction sample, 24 April 2005, K. Edward and S. Callan (WAM T129321); 2 individuals, Winkler sac, 17 May 2005, S. Callan et al. (WAM T129322); Future administration area, $20^{\circ} 47^{\prime} 45^{\prime \prime} \mathrm{S}, 115^{\circ} 27^{\prime} 15^{\prime \prime} \mathrm{E}, 3$ individuals, hand sorted litter 17 May 2005, S. Callan et al. (WAM T129324); 5 individuals, Winkler sac, 7 May 2005, S. Callan et al. (WAM T129327); Plot N 01a, current accommodation, 20 $49^{\prime} 34^{\prime \prime}$, $115^{\circ} 26^{\prime} 44^{\prime \prime} \mathrm{E}, 16$ individuals, suction sample, 1 May 2007, S. Callan and K. Edwards (WAM T129296); Plot N 02, old dump, 20 $47^{\prime} 47^{\prime \prime}$, $115^{\circ} 21^{\prime} 01^{\prime \prime} \mathrm{E}, 1$ individual, Winkler sac, 1 May 2007, S. Callan and 
K. Edwards (WAM T129306); Plot N 04a barge landing, $20^{\circ} 43^{\prime} 29^{\prime \prime} \mathrm{S}, 115^{\circ} 28^{\prime} 20^{\prime \prime} \mathrm{E}, 10$ individuals, suction sample, 1 May 2007, S. Callan and K. Edwards (WAM T114010); 1 individual, wet pitfall traps, 25 April 2007-1 May 2007, S. Callan and K. Edwards (WAM T129311); Plot N 04, barge landing, $20^{\circ} 43^{\prime} 29^{\prime \prime} \mathrm{S}, 115^{\circ} 28^{\prime} 20^{\prime \prime} \mathrm{E}, 2$ individuals, suction sample, 6 May 2006, S. Callan and R. Graham (WAM T126131); Plot N 04b, barge landing, $20^{\circ} 43^{\prime} 44^{\prime \prime} \mathrm{S}, 115^{\circ} 28^{\prime} 23^{\prime \prime} \mathrm{E}, 20$ individuals, suction sample, 1 May 2007, S. Callan and K. Edwards (WAM T129300); Plot N 05a, current airport, front office, $20^{\circ} 51^{\prime} 58^{\prime \prime} \mathrm{S}, 115^{\circ} 24^{\prime} 22^{\prime \prime} \mathrm{E}, 3$ individuals, wet pitfall trap, 25 April-1 May 2007, S. Callan and K. Edwards (WAM T129302); Plot N 05b, current airport, front office, $20^{\circ} 51^{\prime} 58^{\prime \prime} \mathrm{S}, 115^{\circ} 24^{\prime} 22^{\prime \prime} \mathrm{E}, 1$ individual, Winkler sac, 1 May 2007, S. Callan and K. Edwards (WAM T129303); 1 individual, wet pitfall traps, 25 April- 1 May 2007, S. Callan and K. Edwards (WAM T129308); Plot N 06, storage area ( old airport), $20^{\circ} 47^{\prime} 51^{\prime \prime} \mathrm{S}, 115^{\circ} 25^{\prime} 58^{\prime \prime} \mathrm{E}, 2$ individuals, Winkler sac, 6 May 2006, S. Callan and R. Graham (WAM T129334); 2 individuals, suction sample 7, 6 May 2006, S. Callan and R. Graham (WAM T129461); Plot N 06a, old airport, 20 $17^{\prime} 34^{\prime \prime}$ S, $115^{\circ} 25^{\prime} 27^{\prime \prime}$ E, 2 individuals, suction sample, 1 May 2007, S. Callan and K. Edwards (WAM T129307); Plot N 08, terminal tanks, $20^{\circ} 46^{\prime} 45^{\prime \prime} \mathrm{S}, 115^{\circ} 27^{\prime} 43^{\prime \prime} \mathrm{E}, 3$ individuals, Winkler sac, 1 May 2007, S. Callan and K. Edwards (WAM T129295); 3 individuals, wet pitfalls, 25 April-1 May 2007, S. Callan and K. Edwards (WAM T129305); Plot N10, evaporation pit, $20^{\circ} 49^{\prime} 23^{\prime \prime} \mathrm{S}, 115^{\circ} 22^{\prime} 21^{\prime \prime} \mathrm{E}, 3$ individuals, suction sample, 6 May 2006, S. Callan and R. Graham (WAM T129471); Plot N11, former ODE camp, $20^{\circ} 48^{\prime} 52^{\prime \prime} \mathrm{S}, 115^{\circ} 22^{\prime} 32^{\prime \prime} \mathrm{E}, 5$ individuals, suction sample 7, 6 May 2006, S. Callan and R. Graham (WAM T129464); Plot N 12, Osprey camp, $20^{\circ} 49^{\prime} 55^{\prime \prime} \mathrm{S}, 115^{\circ} 25^{\prime} 52^{\prime \prime} \mathrm{E}, 1$ individual, Winkler sac, 1 May 2007, S. Callan and K. Edwards (WAM T129304); 1 individual, suction sample, 1 May2007, S. Callan and K. Edwards (WAM T129312); 4 individuals, suction sample 7, 6 May 2006, S. Callan and R. Graham (WAM T129465); Plot N 14, old incinerator, $20^{\circ} 48^{\prime} 37^{\prime \prime} \mathrm{S}, 115^{\circ} 25^{\prime} 37^{\prime \prime} \mathrm{E}, 30$ individuals, suction sample, 1 May 2007, S. Callan and K. Edwards (WAM T129299); 4 individuals, suction sample 7, 6 May 2006, S. Callan and R. Graham

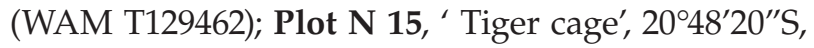
$115^{\circ} 25^{\prime} 52^{\prime \prime} \mathrm{E}, 11$ individuals, suction sample, 1 May 2007, S. Callan and K. Edwards (WAM T129298); 23 individuals, suction sample 7, 6 May 2006, S. Callan and R. Graham (WAM T129459); Plot N16, old pipe dump, 20 $47^{\prime} 51^{\prime \prime} \mathrm{S}, 115^{\circ} 26^{\prime} 56^{\prime \prime} \mathrm{E}, 1$ individual, Winkler sac, 1 May 2007, S. Callan and K. Edwards (WAM T129310); 8 individuals, Winkler sac, 6 May 2006, S.
Callan and R. Graham (WAM T129330); Plot N 17, rehabilitation dump, $20^{\circ} 47^{\prime} 53^{\prime \prime} \mathrm{S}, 115^{\circ} 21^{\prime} 20^{\prime \prime} \mathrm{E}, 4$ individuals, suction sample, 6 May 2006, S. Callan and R. Graham (WAM T129325); Plot N 18, old dump on South Rd., $20^{\circ} 50^{\prime} 29^{\prime \prime}$ S, $115^{\circ} 23^{\prime} 23^{\prime \prime}$ E, 1 individual, wet pitfall traps, 25 April-1 May 2007, S. Callan and K. Edwards (WAM T129297); 89 individuals, suction sample, 8 May 2006, S. Callan and R. Graham (WAM T129315); Plot N19, dump near 'M'station, $20^{\circ} 51^{\prime} 52^{\prime}$ 'S, $115^{\circ} 20^{\prime} 34^{\prime} \mathrm{E}, 2$ individuals, Winkler sac, 6 May 2006, S. Callan and R. Graham (WAM T129473); Plot N20, old air strip, $20^{\circ} 45^{\prime} 00^{\prime \prime} \mathrm{S}, 115^{\circ} 26^{\prime} 51^{\prime \prime} \mathrm{E}, 6$ individuals, suction sample 7, 6 May 2006, S. Callan and R. Graham (WAM T129463); Plot N 22, old drill workshop, $20^{\circ} 49^{\prime} 55^{\prime \prime} \mathrm{S}, 115^{\circ} 25^{\prime} 13^{\prime \prime} \mathrm{E}, 20$ individuals, suction sample, 1 May 2007, S. Callan and K. Edwards (WAM T129301); Plot N26, current gravel pit, $20^{\circ} 49^{\prime} 01^{\prime \prime} \mathrm{S}, 115^{\circ} 26^{\prime} 06^{\prime \prime} \mathrm{E}, 2$ individuals, Winkler sac, 6 May 2006, S. Callan and R. Graham (WAM T129466); Gorgon Project: Footprint plot CC1, $20^{\circ} 49^{\prime} 01^{\prime \prime} \mathrm{S}, 115^{\circ} 26^{\prime} 15^{\prime \prime} \mathrm{E}, 1$ individual, valley flats, wet pitfall traps, 10-15 March 2006, S. Callan and R. Graham (WAM T126137); Footprint plot CC2, $20^{\circ} 49^{\prime} 02^{\prime \prime} \mathrm{S}, 115^{\circ} 26^{\prime} 24^{\prime \prime} \mathrm{E}$, low limestone flats, 1 individual, Winkler sac, 25 September 2006, S. Callan and R. Graham (WAM T129313); 8 individuals, suction sample, 25 September 2006, S. Callan and R. Graham (WAM T129326); 6 individuals, Winkler sac, 15 March 2006, S. Callan and R. Graham (WAM T129455); 3 individuals, suction sample, 25 September 2006, S. Callan and R. Graham (WAM T126125); Footprint plot GP1, $20^{\circ} 47^{\prime} 32^{\prime \prime}$, $115^{\circ} 27^{\prime} 26^{\prime \prime} \mathrm{E}$, coastal dunes, 1 individual, Winkler sac, 15 March 2006, S. Callan and R. Graham (WAM T126138); 4 individuals, suction sample, 15 March 2006, S. Callan and R. Graham (WAM T129467); 1 individual, wet pitfall traps, 1015 March 2006, S. Callan and R. Graham (WAM T129450); Footprint plot GP2, 2047'38"S, $115^{\circ} 27^{\prime} 27^{\prime \prime} \mathrm{E}$, beach and drainage line, 3 individuals, Winkler sac, 15 March 2006, S. Callan and R. Graham (WAM T126132); 3 individuals, wet pitfall traps, 10-15 March 2006, S. Callan and R. Graham (WAM T129329); 3 individuals, suction sample, 15 March 2006, S. Callan and R. Graham (WAM T129460); Footprint plot GP3, 2047'09"S, $115^{\circ} 27^{\prime} 26^{\prime \prime} \mathrm{E}$, floodplain, 3 individuals, suction sample, 15 March 2006, S. Callan and R. Graham (WAM T126134); 1 individual, Winkler sac, 15 March 2006, S. Callan and R. Graham (WAM T126135); Footprint plot GP4, 20 ${ }^{\circ} 47^{\prime} 03^{\prime \prime} S$, $115^{\circ} 27^{\prime} 33^{\prime \prime} \mathrm{E}$, low limestone flats, 8 individuals, Winkler sac, 15 March 2006, S. Callan and R. Graham (WAM T129457); 2 individuals, wet pitfall traps, 10-15 March 2006 S. Callan and R. Graham 
(WAM T129469); 4 individuals, suction sample, 15 March 2006, S. Callan and R. Graham (WAM T129470); Footprint plot GP6, 2047'05"S, $115^{\circ} 26^{\prime} 28^{\prime \prime} \mathrm{E}$, high limestone flats, 3 individuals, Winkler sac, 25 September 2006, S. Callan and R. Graham (WAM T126122); 1 individual, wet pitfall traps, 15 March 2006, S. Callan and R. Graham (WAM T129468); Footprint plot GP7, 2047'51"S, $115^{\circ} 26^{\prime} 27^{\prime \prime} \mathrm{E}$, limestone ridge to drainage line, 1 individual, wet pitfall, 10-15 March 2006, S. Callan and R. Graham (WAM T99491); 1 individual, suction sample, 15 March 2006, S. Callan and R. Graham (WAM T129474); 3 individuals, Winkler sac, 15 March 2006, S. Callan and R. Graham (WAM T129475); Footprint plot GP8, 2047'59"S, $115^{\circ} 26^{\prime} 25^{\prime \prime} \mathrm{E}$, valley flats, 3 individuals, suction sample, 15 March 2006, S. Callan and R. Graham (WAM T129454); 3 individuals, wet pitfall traps, 1015 March 2006, S. Callan and R. Graham (WAM T129456); Footprint plot GP9, 2047'59"S, $115^{\circ} 27^{\prime} 00^{\prime \prime} \mathrm{E}$, low limestone ridge, 5 individuals, suction sample, 15 March 2006, S. Callan and R. Graham (WAM T126124) 1 individual, wet pitfall traps, 20-25 September 2006, S. Callan and R. Graham (WAM T126133); Footprint plot GPX, $20^{\circ} 47^{\prime} 45^{\prime \prime} \mathrm{S}, 115^{\circ} 27^{\prime} 08^{\prime \prime} \mathrm{E}$, low limestone ridge, 4 individuals, suction sample, 25 September 2006, S. Callan and R. Graham (WAM T126123); 5 individuals, Winkler sac, 15 March 2006, S. Callan and R. Graham (WAM T126136); 12 individuals, wet pitfall traps, 10-15 March 2006, S. Callan and R. Graham (WAM T129458); 3 individuals, suction sample, 15 March 2006, S. Callan and R. Graham (WAM T129472).

\section{DIAGNOSIS}

Unixenus mjoebergi differs from other species of the genus in having 3 basiconic sensilla on antennal article VI, biarticulate setae with spiny projections surrounding the central flagellum on the coxae, prefemora and femora, 1 seta on all tibia and 2 on all femora, 3 ornamental trichomes $c$ each side dorsal to the caudal bundle, and 2 pairs of coxal glands.

\section{DESCRIPTION}

See Short and Huynh (2011).

\section{REMARKS}

Unixenus mjoebergi was first described by Verhoeff (1924) based on specimens collected from the Kimberley region of Western Australia, and is now known to occur in many different regions of northeastern and north-western Australia, including Barrow Island (Short and Huynh 2011) (Figures 2, 8).

\section{Family Synxenidae Silvestri, 1923}

\section{Phryssonotus Scudder, 1885}

Lophonotus Menge, 1854: 12 (junior homonym of Lophonotus Stephens, 1829).

Phryssonotus Scudder, 1885: 731 (replacement name for Lophonotus Menge, 1854).

Synxenus Silvestri, 1900: 114 (junior synonym).

Kubanus Attems, 1926: 113 (junior synonym).

Koubanus Attems, 1928: 198 (junior synonym).

Schindalmonotus Attems, 1926: 113 (junior synonym).

\section{TYPE SPECIES}

Lophonotus hystrix Menge, 1854, by original designation.

\section{REMARKS}

The genus Phryssonotus is found globally with six recent and two fossil species (Nguyen DuyJacquemin and Geoffroy 2003). Phryssonotus novaehollandiae is the only Australian species.

\section{Phryssonotus novaehollandiae (Silvestri, 1923)}

Synxenus novaehollandiae Silvestri, 1923: 14, Figure 6.

Phryssonotus novaehollandiae (Silvestri): Condé, 1954: 75.

\section{MATERIAL EXAMINED}

Australia: Western Australia: Barrow Island: 1 individual, 10-15 February 1981, W. H. Butler (WAM $\mathrm{T} 126128) ;$ Bandicoot Bay, $20^{\circ} 52^{\prime} 04^{\prime \prime} \mathrm{S}_{\text {, }} 115^{\circ} 20^{\prime} 01^{\prime \prime} \mathrm{E}$, $40+$ individuals, wet pitfall, 4 November -3 December 1993, M. S. Harvey \& J. M. Waldock (WAM T71103); Cape Malouet, $20^{\circ} 43^{\prime} 03^{\prime \prime} S$, $115^{\circ} 23^{\prime} 42^{\prime \prime} \mathrm{E}, 4$ individuals, under rocks on cliff face, 5 November 1993, M. S. Harvey \& J. M. Waldock (WAM T126127); Current airport, $20^{\circ} 52^{\prime} 01^{\prime \prime} \mathrm{S}$, $115^{\circ} 24^{\prime} 19^{\prime \prime} \mathrm{E}, 1$ individual, suction samples (combined), 17 May 2005, S. Callan et al. (WAM T71110); Future administration area, $20^{\circ} 47^{\prime} 45^{\prime \prime} \mathrm{S}$, $115^{\circ} 27^{\prime} 15^{\prime \prime} \mathrm{E}, 1$ individual, wet pitfall traps, 17-22 May 2006, S. Callan et al. (WAM T129377); 2 individuals, Winkler sac, 17 May 2005, S. Callan et al. (WAM T129387); 'The Acacias' 20 43'45"S, $115^{\circ} 27^{\prime} 45^{\prime \prime} \mathrm{E}, 5$ individuals, litter, hand sorted, 24 April 2005, K. Edward and S. Callan (WAM T129388); 'Valley of the Giants', 20 $42^{\prime} 25^{\prime}$ 'S, $115^{\circ} 25^{\prime} 10^{\prime \prime} \mathrm{E}, 2$ individuals, Winkler sac, 24 April 2005, K. Edward and S. Callan (WAM T129399); 
WAPET camp, $20^{\circ} 49^{\prime} 43^{\prime \prime} S, 115^{\circ} 26^{\prime} 40^{\prime \prime} \mathrm{E}, 7$ individuals, wet pitfall traps, 5 November -3 December 1993, M. S. Harvey \& J. M. Waldock

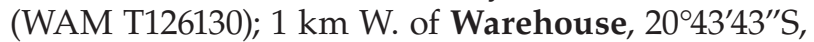
$115^{\circ} 25^{\prime} 56^{\prime \prime} \mathrm{E}, 41$ individuals, wet pitfall traps, 4 November-3 December 1993, M. S. Harvey \& J. M. Waldock (WAM T126129); c. 3 km SW. of Town Point, site V1 (Gorgon Gas Plant Treatment site), $20^{\circ} 47^{\prime} 37^{\prime \prime} \mathrm{S}, 115^{\circ} 26^{\prime} 27^{\prime \prime} \mathrm{E}, 1$ individual, on ground, daytime, 4 July 2008, K. Edwards and N. Gunawardene (WAM T92158); Site 17, 2047'38'S, $115^{\circ} 27^{\prime} 24^{\prime \prime} \mathrm{E}, 1$ individual, wet pitfall traps,17-27 May 2005, S. Callan et al. (WAM T129380); 2 individuals, wet pitfall traps, 24-29 April 2005, K. Edward and S. Callan (WAM T129397); 3 individuals, Winkler sac, 17 May 2005, S. Callan et al. (WAM T129405); Site 22, 2047'12"S, 115²7'17"E, 5 individuals, large wet pitfalls, 24-29 April 2005, K. Edward and S. Callan (WAM T71109); 1 individual, Winkler sac, 17 May 2005, S. Callan et al. (WAM T129395); Site 45, 2047'18"S, $115^{\circ} 26^{\prime} 31^{\prime \prime} \mathrm{E}, 1$ individual, Winkler sac, 17 May 2005, S. Callan et al.

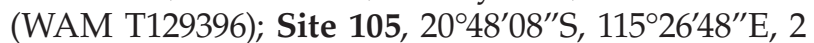
individuals, Winkler sac, 24 April 2005, K. Edward and S. Callan (WAM T129378); 2 individuals, hand sorted litter, 24 April 2005, K. Edward and S. Callan (WAM T129383); 1 individual, Winkler sac, 17 May 2005, S. Callan et al. (WAM T129384); 2 individuals, Winkler sac, 24 April 2005, K. Edward and S. Callan (WAM T129389); SW of Terminal Tanks: site AVN12, 2046'57.4'S, 115²6'46"E, Angusta valley, 1 individual, wet pitfalls, 28 August-1 September 2004, K. Edward and L. Mould (WAM T129342); site ML1A1, 204'15"S, $115^{\circ} 27^{\prime} 09.4^{\prime \prime} \mathrm{E}$, Melaleuca on limestone, 1 individual, wet pitfalls, 28 August-1 September 2004, K. Edward and L. Mould (WAM T129333); 1 individual, site ML1A2, 2047'27.6"S, $115^{\circ} 27^{\prime} 07.5^{\prime \prime} \mathrm{E}$, Melaleuca on limestone, 1 individual, wet pitfalls, 28 August-1 September 2004 K. Edward and L. Mould (WAM T129340); W of town point, site LM1A2, 2047'25.4'S, $115^{\circ} 27^{\prime} 20.7^{\prime \prime} \mathrm{E}$, Triodia on limestone, 1 individual, wet pitfalls, 28 August-1 September 2004, K. Edward and L. Mould (WAM T129332); E of old airport, site TLN13, $20^{\circ} 48^{\prime} 04.3^{\prime \prime} \mathrm{S}, 115^{\circ} 26^{\prime} 32.9^{\prime \prime} \mathrm{E}$, Triodia on loam, 1 individual, wet pitfalls, 28 August-1 September 2004, K. Edward and L. Mould (WAM T129341); Plot N 02, old dump, 20 $47^{\prime} 47^{\prime \prime} S, 115^{\circ} 21^{\prime} 01^{\prime \prime} \mathrm{E}, 6$ individuals, Winkler sac,1 May 2007, S. Callan and K. Edwards (WAM T129351); 4 individuals, Winkler sac, 6 May 2006, S. Callan and R. Graham (WAM T129408); Plot N 04, barge landing, 2043'29"S, $115^{\circ} 28^{\prime} 20^{\prime \prime} \mathrm{E}, 6$ individuals, Winkler sac, 6 May 2006, S. Callan and R. Graham (WAM T129410); Plot N 05, current airport, $20^{\circ} 51^{\prime} 54^{\prime \prime} \mathrm{S}, 115^{\circ} 24^{\prime} 25^{\prime \prime} \mathrm{E}, 4$ individuals, Winkler sac, 6 May 2006, S. Callan and R. Graham (WAM T129415); Plot N 05a, current airport front office, $20^{\circ} 51^{\prime} 58^{\prime \prime} \mathrm{S}, 115^{\circ} 24^{\prime} 22^{\prime \prime} \mathrm{E}, 1$ individual, nocturnal hand collecting, 1 May 2007,
S. Callan and K. Edwards (WAM T129348); 3 individuals, wet pitfall trap, 25 April-1 May 2007, S. Callan and K. Edwards (WAM T129345); Plot N 05b, current airport, helicopter hangar, 20 51'50'S, $115^{\circ} 24^{\prime} 23^{\prime \prime}$, 8 individuals, Winkler sac, 1 May 2007, S. Callan and K. Edwards (WAM T114011); Plot N 06, storage area (old airport), 2047'51'S, $115^{\circ} 25^{\prime} 58^{\prime \prime} \mathrm{E}$, 1 individual, wet pitfall, 1-6 May 2006, S. Callan and R. Graham (WAM T99492); 5 individuals, Winkler sac, 6 May 2006, S. Callan and R. Graham (WAM T129409); Plot N 06a, storage area (old airport), $20^{\circ} 47^{\prime} 34^{\prime \prime} \mathrm{S}, 115^{\circ} 25^{\prime} 27^{\prime \prime} \mathrm{E}, 1$ individual, wet pitfall trap, 25 April-1 May 2007, S. Callan and K. Edwards (WAM T129336); Plot N 06b,1 individual, 1 May 2007, S. Callan and K. Edwards (WAM T129337); Plot N 07, base/warehouse, $20^{\circ} 49^{\prime} 04^{\prime \prime} \mathrm{S}, 115^{\circ} 23^{\prime} 06^{\prime \prime} \mathrm{E}$, 3 individuals, suction sample, 6 May 2006, S. Callan and R. Graham (WAM T129393); Plot N 08, terminal tanks, $20^{\circ} 46^{\prime} 45^{\prime \prime} \mathrm{S}, 115^{\circ} 27^{\prime} 43^{\prime \prime} \mathrm{E}, 3$ individuals, Winkler sac, 6 May 2006, S. Callan and R. Graham (WAM T129416); Plot N 09, central processing facility, $20^{\circ} 47^{\prime} 05^{\prime \prime} \mathrm{S}, 115^{\circ} 23^{\prime} 38^{\prime \prime} \mathrm{E}, 1$ individual, suction sample, 6 May 2006, S. Callan and R. Graham (WAM T129398); Plot N 11, former ODE camp, $20^{\circ} 48^{\prime} 52^{\prime \prime} \mathrm{S}, 115^{\circ} 22^{\prime} 32^{\prime \prime} \mathrm{E}, 1$ individual, wet pitfall trap, 25 April-1 May 2007, S. Callan and K. Edwards (WAM T129339); Plot N 12, Osprey camp, $20^{\circ} 49^{\prime} 55^{\prime \prime} \mathrm{S}, 115^{\circ} 25^{\prime} 52^{\prime \prime} \mathrm{E}, 1$ individual, wet pitfall trap, 25 April-1 May 2007, S. Callan and K. Edwards (WAM T129338); 11 individuals, Winkler sac, 6 May 2006, S. Callan and R. Graham (WAM T129411); 1 individual, wet pitfall trap, 1-6 May 2006, S. Callan and R. Graham (WAM T129414); Plot N 13, old

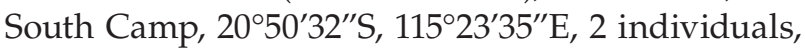
Winkler sac, 6 May 2006, S. Callan and R. Graham (WAM T129413); Plot N 14, old incinerator, $20^{\circ} 48^{\prime} 37^{\prime \prime} \mathrm{S}, 115^{\circ} 25^{\prime} 37^{\prime \prime} \mathrm{E}, 3$ individuals, Winkler sac, 6 May 2006, S. Callan and R. Graham (WAM T129391); Plot N 16, old pipe dump, 2047'51'S, 115 $26^{\prime} 56^{\prime \prime} \mathrm{E}, 2$ individuals, Winkler sac, 6 May 2006, S. Callan and R. Graham (WAM T129390); Plot N 18, old dump on

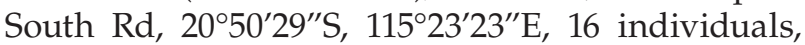
wet pitfall trap, 25 April-1 May 2007, S. Callan and K. Edwards (WAM T129335); 1 individual, nocturnal hand collecting, 1 May 2007, S. Callan and K. Edwards (WAM T129350); 4 individuals, Winkler sac, 1 May 2007, S. Callan and K. Edwards (WAM T129343); 4 individuals, suction sample, 6 May 2006, S. Callan and R. Graham (WAM T129400); Plot N 20, old air strip, 2045'00"S, $115^{\circ} 26^{\prime} 51^{\prime \prime} \mathrm{E}, 1$ individual, suction sample, 6 May 2006, S. Callan and R. Graham (WAM T126120); 2 individuals, Winkler sac, 6 May 2006, S. Callan and R. Graham (WAM T129386); Plot N 23, old administration building, $20^{\circ} 40^{\prime} 09^{\prime \prime} \mathrm{S}, 115^{\circ} 23^{\prime} 40^{\prime \prime} \mathrm{E}, 2$ individuals, Winkler sac, 1 May 2007, S. Callan and K. Edwards (WAM T129347); Plot N 28, near barge landing (CO2 data well site), 20 $47^{\prime} 05^{\prime \prime} \mathrm{S}, 115^{\circ} 23^{\prime} 38^{\prime \prime} \mathrm{E}$, 2 individuals, wet pitfall trap, 1 May 2007, S. Callan 
and K. Edwards (WAM T129344); 1 individual, nocturnal hand collecting, 1 May 2007, S. Callan and K. Edwards (WAM T129346). Gorgon Project:

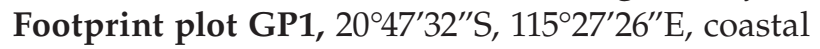
dunes, 1 individual, wet pitfall traps, 21-25 September 2006, S. Callan and R. Graham (WAM T129401); Footprint plot GP3, 2047'09"S, $115^{\circ} 27^{\prime} 26^{\prime \prime} \mathrm{E}$, floodplain, 6 individuals, Winkler sac, 25 September 2006, S. Callan and R. Graham (WAM T129382); Footprint plot GP3, 20 47'09"S, $115^{\circ} 27^{\prime} 26^{\prime \prime} \mathrm{E}$, floodplain, 2 individuals, Winkler sac, 15 March 2006, S. Callan and R. Graham (WAM T129404); 1 individual, wet pitfall traps, 10-15 March 2006, S. Callan and R. Graham (WAM T129406); Footprint plot GP4, 2047'03"S, $115^{\circ} 27^{\prime} 33^{\prime \prime} \mathrm{E}$, low limestone flats, 1 individual, wet pitfall traps, 21-25 September 2006, S. Callan and R. Graham (WAM T129392); 7 individuals, Winkler sac, 15 March 2006, S. Callan and R. Graham (WAM T129407); Footprint plot GP5, 2046'59"S, $115^{\circ} 27^{\prime} 03^{\prime \prime} \mathrm{E}$, high limestone flats, 1 individual, wet pitfall traps, 10-15 March 2006, S. Callan and R. Graham (WAM T129381); 1 individual, 21-25 September 2006, S. Callan and R. Graham (WAM T129394); Footprint plot GP6, 2047'05"S, $115^{\circ} 26^{\prime} 25^{\prime \prime} \mathrm{E}$, high limestone flats, 1 individual, wet pitfall traps, 21-25 September 2006, S. Callan and R. Graham (WAM T129403); Footprint plot GP9, $20^{\circ} 47^{\prime} 59^{\prime \prime} \mathrm{S}, 115^{\circ} 27^{\prime} 00^{\prime \prime} \mathrm{E}$, low limestone ridge, 4 individuals, Winkler sac, 25 September 2006, S. Callan and R. Graham (WAM T129379); 8 individuals, Winkler sac, 15 March 2006, S. Callan and R. Graham (WAM T129412); Footprint plot GPX, $2^{\circ} 47^{\prime} 45^{\prime \prime} \mathrm{S}, 115^{\circ} 27^{\prime} 08^{\prime \prime} \mathrm{E}$, low limestone ridge, 2 individuals, Winkler sac, 15 March 2006S. Callan and R. Graham (WAM T129402).

\section{DIAGNOSIS}

Phryssonotus novaehollandiae can be distinguished from other species of the genus by the presence in adults of eyes with 11 ocelli and 5 frontal trichomes $B$ on each side.

\section{DESCRIPTION}

See Short and Huynh (2006).

\section{REMARKS}

Phryssonotus novaehollandiae was first described as a species of Synxenus by Silvestri (1923) based on specimens collected from Mount Lofty, South Australia. It was redescribed by Short and Huynh (2006) who also documented its occurrence in many different regions of Australia (Short and Huynh 2009), including Barrow Island (Figures 3, 9).

\section{Subclass Chilognatha Latreille, 1802}

\section{Order Spirobolida Cook, 1895}

\section{Family Trigoniulidae Attems, 1909}

\section{Genus Speleostrophus Hoffman, 1994}

Speleostrophus Hoffman, 1994: 20, Figures 1-4.

\section{TYPE SPECIES}

Speleostrophus nesiotes Hoffman, 1994, by original designation.

\section{REMARKS}

The genus is represented by a single troglomorphic species endemic to the subterranean karst ecosystems of Barrow Island (Hoffman 1994).

\section{Speleostrophus nesiotes Hoffman, 1994}

Speleostrophus nesiotes Hoffman, 1994: 20, Figures $1-7$.

\section{MATERIAL EXAMINED}

\section{Holotype}

Australia: Western Australia: Barrow Island: $\mathrm{O}^{\prime}$, Ledge Cave (Cave B1), 20 $47^{\prime} 48^{\prime \prime} \mathrm{S}, 115^{\circ} 19^{\prime} 58^{\prime \prime} \mathrm{E}$, 12 September 1991, W. F. Humphreys and B. Vine (WAM T26696; original number WAM 93/2).

\section{Paratypes}

Australia: Western Australia: Barrow Island: 1 ㅇ, Ledge Cave (Cave B1), 20 $47^{\prime} 48^{\prime \prime} \mathrm{S}, 115^{\circ} 19^{\prime} 58^{\prime \prime} \mathrm{E}$, 5 September 1991, W. F. Humphreys and B Vine (WAM T26697; original number WAM 93/3); 1 , 3 , locality as for holotype, 12 December 1991, D. Goodgame (WAM T26698-T26701; original numbers WAM 93/4-93/7).

\section{Other material examined}

Australia: Western Australia: Barrow Island: $1 \hat{\partial}$, $4.0 \mathrm{~km} \mathrm{~N}$. of Chevron Texaco Camp (B16; T1-1) vial $15,20^{\circ} 47^{\prime} 32^{\prime \prime} \mathrm{S}, 115^{\circ} 27^{\prime} 03^{\prime \prime} \mathrm{E}$, litter trap, 28 February -18 April 2005, L. Mould and G. Humphreys (WAM T66022); 1 ㅇ, $3.0 \mathrm{~km} \mathrm{~N}$. of Chevron Texaco Camp (S6; T1-1) vial 201, 20 $48^{\prime} 04^{\prime \prime} \mathrm{S}, 115^{\circ} 26^{\prime} 44^{\prime \prime} \mathrm{E}$, litter trap, 28 February-18 April 2005, L. Mould and G. Humphreys (WAM T66023); 1 +, 1 juvenile, $1.3 \mathrm{~km}$ NW. of Chevron Texaco Camp (S9; T1-2) vial 62, $20^{\circ} 49^{\prime} 12^{\prime \prime} \mathrm{S}, 115^{\circ} 26^{\prime} 04^{\prime \prime} \mathrm{E}$, litter trap, 28 February-18 April 2005, L. Mould and G. Humphreys (WAM T66024); 2 o, 1 ㅇ, $4 \mathrm{~km} \mathrm{~N}$. of Chevron Texaco Camp (BH-16), 20 $47^{\prime} 32^{\prime \prime} \mathrm{S}, 15^{\circ} 27^{\prime} 03^{\prime \prime} \mathrm{E}$, litter trap, 4 March-19 April 2005, L. Mould and G. Humphreys (WAM T66025); $1 \mathrm{o}, 4 \mathrm{~km} \mathrm{~N}$. of Chevron Texaco

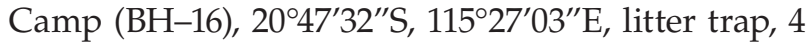
March-19 April 2005, L. Mould and G. Humphreys (WAM T66026); 1 ㅇ $3.0 \mathrm{~km} \mathrm{~N}$. of Chevron Texaco 
Camp (borehole S6; Trap 1-50), 2048'04"S, $115^{\circ} 26^{\prime} 44^{\prime \prime} \mathrm{E}$, litter trap, 18 July 2006, L. Mould and K.L. Edward (WAM T76949); 1 juvenile, 1.3 km NW. of Chevron Texaco Camp (borehole S9; Trap 1-83), $20^{\circ} 49^{\prime} 12^{\prime \prime} \mathrm{S}, 115^{\circ} 26^{\prime} 04^{\prime \prime} \mathrm{E}$, litter trap, 18 July 2006, L. Mould and K.L. Edward (WAM T76950); 1 ภ, 3 ㅇ, $4.0 \mathrm{~km} \mathrm{~N}$. of Chevron Texaco Camp (borehole B16; Trap 1-80), 20 $0^{\circ} 47^{\prime} 32^{\prime \prime} \mathrm{S}, 115^{\circ} 27^{\prime} 03^{\prime \prime} \mathrm{E}$, litter trap, 18 July 2006, L. Mould and K.L. Edward (WAM T76951); 1 +, $5.8 \mathrm{~km} \mathrm{~N}$. of Chevron Texaco Camp (borehole S2; Trap 1-80), $20^{\circ} 46^{\prime} 33^{\prime \prime} \mathrm{S}, 115^{\circ} 26^{\prime} 53^{\prime \prime} \mathrm{E}$, litter trap, 18 July 2006, L. Mould and K.L. Edward (WAM T76952) 1 đै, 3.4 km NNW. of Chevron Texaco Camp (borehole S5; Trap 1-84), 20 $47^{\prime} 54^{\prime \prime} \mathrm{S}, 115^{\circ} 26^{\prime} 05^{\prime \prime} \mathrm{E}$, litter trap, 18 July 2006, L. Mould and K.L. Edward (WAM T76953); 1 , Barrow Island, no other data, $20^{\circ} 49^{\prime} 12^{\prime \prime} \mathrm{S}, 115^{\circ} 26^{\prime} 04^{\prime \prime} \mathrm{E}$, troglofauna trap, $8 \mathrm{~m}, 17$ January 2010, J. Alexander (WAM T110381).

\section{DIAGNOSIS}

Speleostrophus nesiotes differs from other trigoniulids by its pallid colouration and its lack of ocelli (Figure 4).

\section{DESCRIPTION}

See Hoffman (1994).

\section{REMARKS}

Speleostrophus nesiotes is restricted to subterranean karst ecosystems on Barrow Island, where it occurs on both the eastern and western side of the island. On the island, this species was collected mainly from boreholes. These are narrow vertical shafts drilled down from the earth's surface for mining and hydrogeological exploration purposes to depths of 50-800 m underground. Many are open to the surface and unlined (connected to the surrounding substrate). Litter-filled troglofauna traps are suspended in the shafts to attract and collect subterranean fauna (Environmental Protection Authority 2003; Irwin 2007).

The highly restricted distribution of $S$. nesiotes (Figure 10) suggests it is a short-range endemic species (Harvey 2002; Harvey et al. 2011). For this reason, it is listed as threatened under the Western Australian Wildlife Conservation Act 1950.

Order Polydesmida Pocock, 1887

\section{Suborder Polydesmidea Pocock, 1887}

\section{Family Haplodesmidae Cook, 1895}

\section{REMARKS}

A single unidentified specimen of this family has been collected from Barrow Island. Due to our poor understanding of haplodesmids in Western
Australia, we have been unable to assign it to any existing genus.

\section{Genus and species indet.}

\section{MATERIAL EXAMINED}

Australia: Western Australia: Barrow Island: 1

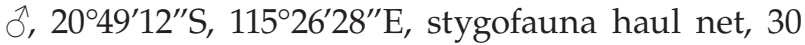
April 1994, J. Alexander (WAM T116231).

\section{REMARKS}

All Haplodesmidae lack eyes and most family members are less well pigmented and sclerotized than other polydesmidan families. The specimen from Barrow Island (Figure 5, 11) was found underground, lacks pigment and has relatively long legs, all troglomorphic features. Golovatch et al. (2009) comment, however, that very few haplodesmids, and only those with 'soft integuments', could be described as troglobites, but even their status would be uncertain. Although this specimen represents a new species, more information is needed before it can be considered a troglobite.

\section{Family Paradoxosomatidae Daday, 1889}

\section{Subfamily Australiosomatinae Brölemann, 1916}

\section{Genus Boreohesperus Shear, 1992}

Boreohesperus Shear, 1992: 778.

TYPE SPECIES

Boreohesperus capensis Shear, 1992, by original designation.

\section{REMARKS}

The genus Boreohesperus was described for a single species from Cape Range, Western Australia (Shear 1992). In a recent review of the genus (Car and Harvey 2013), a further five species were described, one endemic to Barrow Island, four from mainland Australia.

\section{Boreohesperus dubitalis Car and Harvey, 2013}

Boreohesperus dubitalis Car and Harvey, 2013: 10-13, Figures 1D, 5, 6, 9.

\section{MATERIAL EXAMINED}

See Car and Harvey (2013).

\section{REMARKS}

This species is restricted to Barrow Island where it is fairly abundant and widespread (Figures 6, 12). 


\section{DISCUSSION}

The millipede fauna of Barrow Island consists of just six species. Two of the three species of pin-cushion millipedes, Unixenus mjoebergi and Phryssonotus novaehollandiae, are known to be found elsewhere in Australia and are not endemic to Barrow Island. They have a wide although patchy distribution under bark and in leaf litter and soil habitats (Short and Huynh 2009, 2011). The third pin-cushion millipede, Lophoturus madecassus, is newly recorded from Australia, but has a wide, circum-tropical distribution including Madagascar, Tonga, Cook Islands, Algeria, Ivory Coast, Jamaica, Florida, and Cape Verde Islands.

There is one confirmed troglobitic species on the island, Speleostrophus nesiotes and a new species of Haplodesmidae that is probably troglobitic and also most likely represents a new genus. Both are restricted to subterranean habitats on Barrow Island.

The epigean millipede Boreohesperus dubitalis is also restricted to Barrow Island where it is widespread and abundant. Other species of the genus occur on adjacent areas of mainland Australia, including the Cape Range and the Pilbara bioregion (Shear 1992; Car and Harvey 2013).

\section{ACKNOWLEDGEMENTS}

We wish to thank Paul West (Cliffs Natural Resources) and Stephen White (BHP Billiton Iron Ore) for the financial support that allowed CAC to undertake this project. We also thank the many collectors who persevered in collecting millipedes on the island, in particular Julianne Waldock, Jason Alexander, Shae Callan, Jonathan Majer, Bill Humphreys, Karen Edward, Roy Teale, and Garth Humphreys. We also thank Julianne Waldock for assistance in the laboratory.

\section{REFERENCES}

Attems, C. (1926). Myriopoda (pp. 1-402). In: Kükenthal, W. and Krumbach, T. (eds), Handbuch der Zoologie, vol. 4(1). Walter de Gruyter and Co.: Berlin and Leipzig.

Attems, C. (1928). The Myriapoda of South Africa. Annals of the South African Museum 26: 1-431.

Brölemann, H.W. (1931). Myriapodes recueillis par Mme Pruvot en Nouvelle-Caledonie et aux Loyalty. Archives de Zoologie Experimentale et Generale, serie $V$ 72: 275-316.

Car, C.A. and Harvey, M.S. (2013). A review of the Western Australian keeled millipede genus Boreohesperus (Diplopoda: Polydesmida: Paradoxosomatidae) Zookeys 290: 1-19.

Chamberlin, R.V. (1955). New millipeds from Peru and adjacent parts. University of Utah, Biological Series 11(5): 1-47.
Condé, B. (1954). Les diplopodes Pénicillates de l'ambre et la faune actuelle. Bulletin de la Société Zoologique de France 79: 74-78.

Condé, B. and Nguyen Duy-Jacquemin, M. (1977). De l'utilisation nouvelle du nom de genre Lophoturus Brolemann (Diplopodes, Penicillates). Bulletin $d u$ Museum National d'Histoire Naturelle, Paris, serie 3 477: 90.

Department of Resources, Energy and Tourism (2010). Regional Geology of the Northern Carnarvon Basin. Offshore Petroleum Exploration Acreage Release Report. Australian Government.

Enghoff, H. (1993). Cape Verdean millipedes (Diplopoda). Tropical Zoology 6(1): 207-216.

Environment Australia (2000). Revision of the Interim Biogeographic Regionalisation for Australia (IBRA) and Development of Version 5.1. Environment Australia: Canberra.

Golovatch, S.I., Geoffroy, J.-J., Mauriès, J.-P. and VandenSpiegel, D. (2009). Review of the millipede family Haplodesmidae Cook, 1895, with descriptions of some new or poorly-known species (Diplopoda, Polydesmida). In: Golovatch, S. I.; Mesibov, R. (eds.) Advances in the systematics of Diplopoda I. Zookeys 7: $1-53$.

Harvey, M.S. (2002). Short-range endemism in the Australian fauna: some examples from non-marine environments. Invertebrate Systematics 16: 555-570.

Harvey, M.S., Rix, M.G., Framenau, V.W., Hamilton, Z.R., Johnson, M.S., Teale, R.J., Humphreys, G. and Humphreys, W.F. (2011). Protecting the innocent: studying short-range endemic taxa enhances conservation outcomes. Invertebrate Systematics 25: $1-10$.

Hoffman, R.L. (1994). Studies on spirobolid millipeds. XVIII. Speleostrophus nesiotes, the first known troglobitic milliped, from Barrow Island, Western Australia (Spirobolida: Pachybolidae: Trigoniulinae). Myriapodologica 3: 19-24.

Hoffman, R.L. (1999). Checklist of the millipeds of North and Middle America. Virginia Museum of Natural History, Special Publication 8: 1-584.

Humphreys, W.F. (2000). The hypogean fauna of the Cape Range Peninsula and Barrow Island, northwestern Australia (pp. 581-601). In: Wilkens, H., Culver, D.C. and Humphreys, W.F. (eds), Ecosystems of the world, vol. 30: Subterranean ecosystems, Elsevier: Amsterdam.

Humphreys, W.F. (2001). The subterranean fauna of Barrow Island, northwestern Australia, and its environment. Mémoires de Biospéologie 28: 107-127.

Irwin, R. (2007). Hydrogeology of the Dongara Borehole Line. Department of Water, Hydrogeological Record Series HG4, Government of Western Australia.

Jeekel, C.A.W. (1963). Alloproctinus nom. nov. (Diplopoda, Pollyxenida). Entomologische Berichten 23: 156.

Jones, S. (1937). On two new South Indian pselaphognathous diplopods. Zoologischer Anzeiger 119: 138-146.

Jones, S. (1944). Mechanism of defence in a pselaphognathous diplopod, Unixenus padmanabhii Jones [Abstract] (pp. 94-95). In: Proceedings of the Indian Science Congress, vol. 31(3). Asiatic Society of Bengal, Asiatic Society: Calcutta. 
Marquet, M.L. and Condé, B. (1950). Contribution a la connaissance des diplopodes penicillates d'Afrique et de la region Madecasse. Memoires de l'Institut Scientifique de Madagascar 4(1): 113-134.

Mesibov, R. (2013). Millipedes of Australia. R. Mesibov. http://www.polydesmida.info/ millipedesofaustralia/index.html. Accessed 10 February 2013.

Nguyen Duy-Jacquemin, M. and Condé, B. (1982). Lophoproctides insulaires de l'ocean Pacifique (Diplopodes: Penicillates). Bulletin du Muséum National d'Histoire Naturelle, Paris 4: 95-118.

Nguyen Duy-Jacquemin, M. and Geoffroy, J.-J. (2003). A revised comprehensive checklist, relational database, and taxonomic system of reference for the bristly millipedes of the world (Diplopoda, Polyxenida). African Invertebrates 44: 89-101.

Shear, W.A. (1992). A new genus and two new species of millipedes from the Cape Range, Western Australia (Diplopoda, Polydesmida, Paradoxosomatidae). Records of the Western Australian Museum 15: 777-784.

Short, M. and Huynh, C. (2006). Redescription of Phryssonotus novaehollandiae (Silvestri, 1923) with details of post-embryonic stadia. Norwegian Journal of Entomology 53: 211-222.

Short, M. and Huynh, C. (2009). Phryssonotus novaehollandiae Silvestri, 1923: the sole Australian representative of the millipede family Synxenidae. Soil Organisms 81: 695-700.

Short, M. and Huynh, C. (2011). The genus Unixenus Jones, 1944 (Diplopoda, Penicillata, Polyxenida) in Australia. Zookeys 156: 105-122.

Short, M. and Huynh, C. (2013). Four new species of Unixenus Jones, 1944 (Diplopoda, Penicillata, Polyxenida) from Australia. Zookeys 278: 75-90.

Silvestri, F. (1923). Notizia della presenza del genere Synxenus (Myriapoda, Diplopoda) in Catalogna e descrizione di quattro specie. Treballs del Museu de Ciències Naturals de Barcelona 4(5): 5-15.

Silvestri, F. (1948). Tavola sinottica dei generi dei Diplopoda Penicillata. Bolletino del Reale Laboratorio di Entomologia Agraria di Portici 8: 214-220.

Thackway, R. and Cresswell, I.D. (eds) (1995). An Interim Biogeographic Regionalisation for Australia: a framework for establishing the national system of reserves, Version 4.0. Australian Nature Conservation Agency: Canberra.

Verhoeff, K.W. (1924). Results of Dr. E. Mjöberg's Swedish Scientific Expeditions to Australia 1910-1913. 34. Myriapoda: Diplopoda. Arkiv för Zoologi 16(5): 1-142.

MANUSCRIPT RECEIVED 16 APRIL 2013; ACCEPTED 30 APRIL 2013. 